\title{
Teleparallel gravity as a gauge theory: coupling to matter with Cartan connection
}

\author{
E. Huguet ${ }^{1}$, M. Le Delliou ${ }^{2,3}$, M. Fontanini ${ }^{1}$, and Z.-C. Lin ${ }^{4}$ \\ 1 - Université de Paris, APC-Astroparticule et Cosmologie (UMR-CNRS 7164), F-75006 Paris, France.* \\ 2 - Institute of Theoretical Physics, School of Physical Science and Technology, \\ Lanzhou University, No.222, South Tianshui Road, Lanzhou, Gansu 730000, P R China \\ 3 - Instituto de Astrofísica e Ciências do Espaço, Universidade de Lisboa, \\ Faculdade de Ciências, Ed. C8, Campo Grande, 1769-016 Lisboa, Portugal ${ }^{\dagger}$ and \\ 4 - Institute of Theoretical Physics 83 Research Center of Gravitation, Lanzhou University, China $\ddagger$
}

(Dated: September 1, 2020)

\begin{abstract}
We present a consistent and complete description of the coupling to matter in the Teleparallel Equivalent to General Relativity (TEGR) theory built from a Cartan connection, as we proposed in previous works. A first theorem allows us to obtain parallel transport from the Cartan connection into a proper Ehresmann connection, while a second ensures to link the TEGR-Cartan connection to the Ehresmann one-form that contains the Levi-Civita connection. This yields a coupling to matter in agreement with observations and the Equivalence Principle. As the fundamental fields proceed from the Cartan connection, if one insists on interpreting TEGR as a gauge theory of translations, such translation gauge field can be extracted from the consistent theory presented. However, this would entail a fundamental change in the structures known for gauge theory and a split between gauge field and connection is imperative. The willingness to take such a step is left to the reader.
\end{abstract}

PACS numbers: 04.50.-h, 11.15.-q, 02.40.-k

\section{CONTENTS}

I. Introduction

II. Which connection for TEGR?
A. About Cartan connection
B. Cartan connection in TEGR

III. The coupling to matter: overview

IV. The coupling to matter: details
A. The Sharpe theorem
B. Levi-Civita coupling from Weitzenböck one-form
C. The $A M \longrightarrow O M$ map, and the covariant derivative

V. Viewing TEGR as a gauge theory of translations ?6
A. Gauge field vs. connection
B. A new gauge paradigm for TEGR ?

VI. Conclusion

Acknowledgements

A. Relating Ehresmann and Cartan connections

B. Associated bundle $Q$

References

\footnotetext{
* michele.fontanini@gmail.comhuguet@apc.univ-paris7.fr

† (delliou@lzu.edu.cn,)morgan.ledelliou.ift@gmail.com

$\ddagger$ linzch12@lzu.edu.cn
}

1

\section{INTRODUCTION}

In this paper we describe how a coupling to matter can be obtained in a new mathematical framework for the Teleparallel Equivalent to General Relativity (TEGR) using a reductive Cartan connection. We start from the Levi-Civita connection, or its corresponding one-form in the Cartan (tetrads) formalism, to describe the coupling to matter fields, which, from observational grounds, is known to describe it well.

TEGR is the well known theory in which the effect of gravity does not manifest itself, as in General Relativity (GR), through induced curvature to spacetime, but by instead giving it torsion. Although being equivalent to GR, this approach yields both an alternative description and interpretation to gravity. It has been presented with various perspectives: in Ref. [1] for its translation-gauge presentation, in Ref. [2] for the "pure tetrads formalism" approach, while in Ref. [3], tensorial formalism is used to present it, together with another gravitation equivalent to GR, first introduced in Ref. [4], the Symmetric TEGR (STGR). TEGR has also provided a stout base for modified gravities, such as $f(T)[5,6]$ theories, further generalised with $f(R, T)[7], f(T, B)[8]$. Additional important generalisation comprise Conformal TEGR $[2,9,10]$, or Teleparallel Equivalent to Lovelock Gravity [11], which are also actively studied.

Although the familiar tensorial formalism can be used to formulate TEGR [as in 3], its presentation as a gauge theory for the translation group [see 1, 12, and references herein] allows one to obtain the torsion as the curvature of a connection defined in the principal bundle of translations. Recently, some of the authors pointed out a difficulty in this formalism: the connection oneform is implicitly identified with another mathematical 
object required to define the torsion, the so-called canonical one-form. This one-form is only defined in the bundle of frames and is not a connection, two properties turning the identification problematic [13-15].

The idea of relating TEGR, and, more generally, gravity, to translations is a very natural one, since the Noether current associated with them is the energymomentum tensor. On another hand, extending that link to a gauge theory of the translations group could be more questionable. Indeed, gauge theories are very successful in the standard model of particle physics describing fundamental interactions, except gravity, in Minkowski space. There, the gauges groups $(U(1), S U(2)$, etc...) only act on fields, not on spacetime (their action is often said to be "internal"). The aim of these theories is to implement the local invariance of a matter equation (usually the Dirac equation), under their respective symmetry group. This is achieved by introducing gauge fields which couple minimally to matter fields. In that context, local invariance means that the action of the symmetry group on matter fields depends on the position in spacetime.

In gravity the group of invariance involved is the group of local diffeomorphisms of $\mathbb{R}^{4}(\mathrm{GL}(4, \mathbb{R}))$, that is the changes of coordinates mapping spacetime. Its appearence is motivated by the Equivalence Principle (EP) translated operationally into the General Covariance Principle (GCP). In the Cartan (tetrads) formulation, any spacetime tensor is mapped by tetrads to a Lorentz tensor. ${ }^{1}$ Thus, all fields, except the tetrads themselves, are viewed as scalars under changes of coordinates. These fields also belong to representations of the Lorentz group. Indeed, using the appropriate representation of the Lorentz group, spinorial fields can also be taken into account, which is a central feature of the tetrad approach. In this formalism the GCP translates into two invariances: the invariance as a scalar under the change of coordinates, and the invariance under the local Lorentz transformations in the corresponding representation [16]. In this tetrad framework, the coupling of matter fields to gravity is usually obtained by the minimal coupling prescription, where the partial derivative are replaced by the covariant Fock-Ivanenko derivative. In this derivative the coupling term is the spin (or Lorentz) connection, a oneform valued in the Lorentz Lie algebra $\mathfrak{s o}(1,3)$. Then, from a gauge theory perspective, if that coupling can be associated with a local Lorentz invariance, the relation with the local diffeomorphism invariance (already satisfied) is not that obvious. Moreover, the status of tetrads, as representing the gravitational field, is, in a gauge theory framework, unclear.

Indeed, a large amount of works since the sixties has been done to clarify this situation ${ }^{2}$. Different the-

\footnotetext{
1 This corresponds to the usual change of indices: $T_{\ldots \ldots} \ldots \ldots(x)=$ $e_{\mu}^{a}(x) e_{b}^{\nu}(x) T_{\ldots \nu \ldots} \ldots(x)$.

2 To illustrate how that point has been already recognized since the
}

ories, using different symmetry group $\left(\mathbb{R}^{4}\right.$, Poincaré, $\left.\mathrm{GL}(4, \mathbb{R}) \rtimes \mathbb{R}^{4}, \mathrm{SO}(1,4), \ldots\right)$, not limited to the diffeomorphism group $(\mathrm{GL}(4, \mathbb{R}))$, have been obtained. A comprehensive review of this gauge approach is [18].

We will not directly address here this gauge problem in its generality. Instead, we will describe a mathematical framework for TEGR in which the diffeomorphism invariance is canonically satisfied. In this framework, the minimal coupling, through the Levi-Civita connection one-form, is consistent with current observations, and derives from a specific Cartan connection, chosen such that curvature is the torsion. In the tetrad formalism, this amounts to retrieving the usual Fock-Ivanenko covariant derivative. Such derivation from a connection is suitable from a gauge theory perspective of TEGR. This basis will enable us to map out the modifications required on the established framework of gauge theories, if we insist on interpreting our result as a gauge theory for translations: mainly, the dissociation of the gauge field from a connection, and the restriction to the translation "gauge" group, appearing only through its algebra.

The structure of the paper is as follows: Sec. II reviews the motivations in the choice of a reductive Cartan connection; Sec. III summarizes the relevant issues involved in coupling gravity to matter with a Cartan connection and gives a setup for the derivation of that coupling to matter; technical details are the subject of Sec. IV; we then discuss Sec. V, the extensions of the gauge paradigm which could be considered in order to interpret TEGR, obtained from our results, as a gauge theory for translations; we finally conclude Sec. VI; some complements on technical details are given in appendices.

For general notions and definitions regarding differential geometry we refer the Reader to [19-22]

\section{WHICH CONNECTION FOR TEGR?}

In this section we remind our motivation in using a Cartan connection to describe TEGR. For the sake of completeness let us first recall some defining properties of the Cartan connection. More detailed account in the context of gravity may be found in [23, 24], while a comprehensive mathematical reference is [25].

\section{A. About Cartan connection}

Let us first recall some facts about Ehresmann connections on principal $G$-bundle (a principal fiber bundle of

birth of gauge theories let us quote the introduction of a review of 1985 by Ivanenko and Sardanashvily [17, p. 4]: "The main dilemma which during 25 years has been confronting the establishment of the gauge gravitation theory, is that gauge potentials represents connections on fiber bundles, while gravitational fields in GR are only metric or tetrad (vierbein) fields." 
Lie group $G$ ). Each tangent space on a point of the fiber bundle contains a vertical subspace defined as the tangent space of the fiber at this point. Any complementary subspace of this vertical space is said to be horizontal. An Ehresmann connection defines in a unique way the notion of horizontality in a principal $G$-bundle: it specifies horizontal subspaces. This is usually done through a connection one-form $\omega_{E}$ whose kernel defines a horizontal space at each point of the total space. Such a one-form is defined by the following properties:

1. it takes its values in the Lie algebra $\mathfrak{g}$ of the Lie group $G$,

2. it is G-invariant: $R_{g}^{*} \omega=A d_{g^{-1}} \omega, R_{g}$ being the right action of $G$ on the bundle,

3. it reduces to the Maurer-Cartan form $\omega_{G}$ of the group $G$ along the fibers: $\omega_{E}(V)=\omega_{G}(V)$, for any vertical vector $V$.

Now, let us recall the definition properties of a Cartan connection on a principal $H$-bundle, $H$ being a subgroup of a Lie group $G$. The Cartan connection is defined through the one form $\omega_{C}$ such that:

1. it takes values in the algebra $\mathfrak{g} \supset \mathfrak{h}$ of $G \supset H$.

2. it is H-invariant: $R_{h}^{*} \omega=A d_{h^{-1}} \omega, R_{h}$ being the right action of $H$ on the bundle,

3. it reduces to the Maurer-Cartan form $\omega_{H}$ of the group $H$ along the fibers,

4. it is, at each point $p$ of the $H$-bundle, a linear isomorphism between the tangent space $T_{p} P$ at $p$ and the Lie algebra $\mathfrak{g}$. This property requires that $G$ has the same dimension as the tangent space $T_{p} P$.

The properties (1) and (4) distinguish the Cartan connection from Ehresmann's. As a consequence of the above properties, the tangent space of the base manifold $M$ can be locally identified with the tangent space $\mathfrak{g} / \mathfrak{h}$ of the homogeneous space ${ }^{3} G / H$. Indeed, the condition (4) precisely states that the $H$-principal bundle is soldered to its base $M$.

For a $(3+1)$-dimensional manifold there are only three possible homogeneous spaces: both (Anti)-de Sitter spaces, and the Minkowski space. The corresponding Cartan geometries have the property of being reductive [see 25, p197 for a definition], implying that, the Cartan connection takes the form

$$
\omega_{C}=\omega+\theta
$$

3 Note that both $G / H$, with $H$ a closed subgroup of $G$, being a homogeneous space, and the fact that $\mathfrak{g} / \mathfrak{h}$ can be identified with its tangent space are known results of differential geometry of Lie groups (see for instance [19] p. 294 for the former statement, and $[25]$ p. 163 , for the latter). where $\omega$ is an $\mathfrak{h}$-valued connection one-form of Ehresmann type, and $\theta$ a $\mathfrak{g} / \mathfrak{h}$-valued one-form, both defined on the principal $H$-bundle. The reductive property also imply that the curvature of the Cartan connection splits into two parts:

$$
\Omega\left(\omega_{C}\right)=\Omega(\omega)+\Theta(\omega)
$$

where $\Omega(\omega)=d \omega+\omega \wedge \omega$ is the curvature of the connection $\omega$ and $\Theta(\omega)=d \theta+\omega \wedge \theta$ its torsion.

\section{B. Cartan connection in TEGR}

In previous works $[13,15]$ we found that one encounters difficulties in the formulation of TEGR as a gauge theory of translations, mainly because of the problematic implicit identification of the Ehresmann translation connection, defined in a principal translation bundle $\left(\mathbb{R}^{4}\right.$ bundle), with the canonical one-form $\theta$ appearing in the definition of torsion: $\Theta(\omega)=d \theta+\omega \wedge \theta$, and which is not an Ehresmann connection. The $\mathbb{R}^{4}$-valued one-form $\theta$ is defined in the bundle of frames $L M$, a principal $G L(4, \mathbb{R})$ bundle, and its sub-bundle of orthonormal frames $O M$, a principal $\mathrm{SO}(1,3)$-bundle, through

$$
(\theta(e), V)=\left(e^{-1}, \pi_{*} V\right),
$$

where $e$ is a frame in $L M$ over a point $x$ of the base manifold $M, V$ a vector of $T L M$, and $\pi$ the projection on the base. In coordinates the above relation reads: $\theta^{a}(e)[V]=e^{a}\left[\pi_{*} V\right]=V^{a}$.

In an attempt to remedy this situation, we proposed to consider a Cartan connection directly defined over the bundle of orthonormal frames $O M$. The choice of a Cartan connection is first of all motivated by the fact that, when defined on $O M$, it can be chosen such that its curvature is the torsion, a central property for TEGR. This is related to the property for the Cartan connection on $O M$ to be reductive (see Sec. II A). In this case the curvatureless and torsion-full Cartan connection one-form reads

$$
\omega_{C}=\omega_{W}+\theta
$$

where the Ehressman term, $\omega_{W}$, is the curvature-less Weitzenböck one-form connection and where the term, $\theta$, coincides with the canonical one-form on $O M$.

We remark that the term $\theta$ of the Cartan connection (2) takes its values precisely in the translation subalgebra $\mathfrak{g} / \mathfrak{h}=\mathbb{R}^{4}$ of the Poincaré algebra, thus local translations are implemented in the Cartan connection although $\theta$ is not a connection by itself.

\section{THE COUPLING TO MATTER: OVERVIEW}

This section aims to summarize how we obtain the Levi-Civita covariant derivative from the reductive Cartan connection we proposed as a possible connection to describe TEGR as a gauge theory. 
The connections usually used in gauge theories are of Ehresmann type. They are associated to parallel transport and covariant derivative. These two notions are not directly available for a Cartan connection. Therefore, to obtain a covariant derivative from a Cartan connection leads to associate it to an Ehresmann connection. An obvious way to realize this mapping in the case of a reductive Cartan connection, is to define parallel transport through the Ehresmann part of the connection. In our context, this part is the Weitzenböck connection $\omega_{W}$, which obviously cannot give the Levi-Civita coupling ${ }^{4}$. We thus have to consider a different way to implement parallel transport. Remark that, the use of the Weitzenböck connection for the parallel transport would imply that the spacetime manifold be parallelizable which correspond to a trivial bundle of frames - and thus the theory not strictly equivalent to $\mathrm{GR}^{5}$. Another, more general, way to build a covariant derivative from a Cartan connection is to use a theorem (proven by R. Sharpe in [25] and reproduced in Appendix A) which, essentially, gives a map between the set of Cartan connections and that of Ehresmann connections satisfying a technical condition (detailed in Sec. IV A). We will, in Sec. IV B, make use of this theorem to map the Cartan connection $\omega_{C}$ defined on the orthonormal frame bundle $O M$ to an affine connection on the principal Poincaré bundle $A M$ (the affine extention of $O M$ viewed as a principal Lorentz bundle). The specialization to the orthonormal bundle, which can be related to the Equivalence Principle, allows us to introduce the contorsion one-form in order to obtain a Levi-Civita connection as the Lorentz part of the affine (Poincaré) connection (this is detailed in Sec. IV B). This affine connection is then mapped back onto the orthonormal frame bundle $O M$ where it divides into two parts: the Levi-Civita connection $\omega_{L C}$ and the canonical one-form $\theta$. However, the Levi-Civita connection obtained that way, appears as the combination of the Weitzenböck connection and its related contorsion $\kappa_{W}$, namely $\omega_{L C}=\omega_{W}-\kappa_{W}$, a well known result, reformulated in the case of fiber bundle in Ref. [28, theorem 6.2.5 p. 79]. Thus, we finally recover the Levi-Civita coupling to matter under the form obtained in [1], opening the possibility to relate this connection to (local) translations.

\section{THE COUPLING TO MATTER: DETAILS}

In this section we detail our proposal to obtain the Levi-Civita covariant derivative from the reductive Cartan connection (2). Our approach mainly relies on a theorem, hereafter referred as Sharpe theorem relating Car-

\footnotetext{
4 Which would respect observational evidence and the Equivalence Principle.

5 Note, however that the solutions exculded from GR by the parallelizability are discarded on physical basis [26, 27].
}

tan and Ehresmann connections. This theorem is proved in [25] and reproduced in Appendix A. We first examine this theorem, and then show how to switch from the Weitzenböck to the Levi-Civita connection in the Lorentz sector. Finally, we use these properties to obtain the covariant derivative on the spacetime manifold.

\section{A. The Sharpe theorem}

Let us first consider the main ingredients of the Sharpe theorem (theorem A.1) and how it specializes in our context. The theorem is concerned by a principal $H$-bundle $P(M, H)$ and its so-called $G$-extension ${ }^{6}$ : the principal $G$-bundle $Q(M, G)=P \times_{H} G$. In our framework, a bundle formalism for TEGR with a Cartan connection [13], $P$ is the bundle of orthonormal frames $O M$ identified with the principal Lorentz $\left(S O_{0}(1,3)\right)$-bundle, and $Q$ is the Poincaré-bundle $A M$, its corresponding affine bundle with structure group $\mathcal{P}:=S O_{0}(1,3) \rtimes \mathbb{R}^{4}$.

A central assumption of the theorem is the existence of an $H$ bundle-map. $\varphi: P \longrightarrow Q$, that is a continuous map such that $\varphi(p h)=\varphi(p) h$ with $p \in P$ and $h \in H \subset G$. The theorem states that $\varphi^{*}$ is a one-to-one correspondence between the set of Ehresmann connections on $Q$ (here $A M$ ), whose kernel does not contains non-null vectors of $\varphi_{*}(T P)$ (the technical condition we mention in Sec. III), and the set of Cartan connections on $P$ (here $O M)$.

Our goal is to obtain explicitly the expression, on a Cartan connection one-form $\omega_{C}$, of the inverse map of $\varphi^{*}$, that is $\left(\varphi^{*}\right)^{-1}\left(\omega_{C}\right)$. In theorem A.1, $\left(\varphi^{*}\right)^{-1}$ isn't formulated in a closed form. Instead, it is obtained as the extension of a general Cartan connection $\omega_{C}$ to a one-form on the product $P \times G: j\left(\omega_{C}\right)$, in Eq. (A1). This one-form $j\left(\omega_{C}\right)$ is proven to be the pull-up from $Q$ to $P \times G$ of an Ehresmann connection $\omega_{E}$ whose kernel intersection with $\varphi_{*}(T P)$ is reduced to zero, that is precisely the image of $\omega_{C}$ by $\left(\varphi^{*}\right)^{-1}$.

An explicit expression of $\omega_{E}=\left(\varphi^{*}\right)^{-1}\left(\omega_{C}\right)$ for a Cartan connection $\omega_{C}$ requires to specify Eq. (A1) in a local trivialization.

We first recast it for our matrix Lie group:

$$
j\left(\omega_{C}\right)=g^{-1} \omega_{C} g+g^{-1} d g,
$$

taking into account the projections appearing in Eq. (A1) by recalling, for a product of manifolds $M \times N$, that one can always write $T_{(m, n)}(M \times N)=T_{m} M \oplus T_{n} N$. In this form, $\omega_{C}$ acts on $T P$ and $g^{-1} d g$, the Maurer-Cartan form of $G$, acts on $T G$.

To simplify matters, let us specify a local trivialization $-f_{a}: \pi^{-1}\left(U_{a}\right) \longrightarrow U_{a} \times F$, where $\pi$ is the projection on the base, $\left\{U_{a}\right\}$ an open covering of $M$ and $F$ a fiber -

\footnotetext{
${ }^{6}$ see for instance [20] and Appendix B.
} 
by the local product $U \times F$ for some open set $U$ of the covering. We then set

$$
\begin{aligned}
& P_{U}:=U \times H, \\
& Q_{U}:=U \times G,
\end{aligned}
$$

and accordingly: $(P \times G)_{U}=P_{U} \times G$. Local coordinates for points $p \in P_{U}, q \in Q_{U}$ will be hereafter denoted by $p=(x, h)$ and $q=(x, g)$ respectively, with $x \in U$.

In a local trivialisation corresponding to $U \times G, G$ being a matrix Lie group, a connection one-form $\omega$ can be decomposed as:

$$
\omega(x, g)=g^{-1} \omega^{U}(x) g+g^{-1} d g,
$$

where $\omega^{U}(x)$ acts on tangent vectors of $T_{x} U \subset T_{x} M$ and $g^{-1} d g$ is the Maurer-Cartan form acting on vectors of $T_{g} G$. Applying Eq. (4) to the Cartan connection $\omega_{C}$ in the trivialisation corresponding to $P_{U}$ gives

$$
\omega_{C}(x, h)=h^{-1} \omega_{C}^{U}(x) h+h^{-1} d h .
$$

Inserting Eq. (5) in Eq. (3) gives the expression of $j\left(\omega_{C}\right)$ in the local trivialization corresponding to $P_{U} \times G$ :

$$
\begin{aligned}
\left(j\left(\omega_{C}\right)\right)(x, h, g) & =g^{-1}\left(h^{-1} \omega_{C}^{U}(x) h+h^{-1} d h\right) g \\
& +g^{-1} d g,
\end{aligned}
$$

In the trivializations defined above, the mapping from $P \times G$ to $Q$, which allows us to relate $j\left(\omega_{C}\right)$ to $\omega_{E}$, can be obtained by setting the coordinate on the fiber $H$ of $P_{U}$ to the neutral element $e$ and identifying the result with $Q_{U}$ :

$$
U \times\{e\} \times G=Q_{U} .
$$

This corresponds to the quotient operation performed in defining $Q$ as the associated bundle $P \times{ }_{H} G$ (see Appendix $\mathrm{B}$ for details). Performing this quotient in Eq. (6) gives $\left(\varphi^{*}\right)^{-1}\left(\omega_{C}\right)$, the Ehresmann connection we are looking for, in the local trivialization corresponding to $Q_{U}$ :

$$
\omega_{E}(x, g)=g^{-1} \omega_{C}^{U}(x) g+g^{-1} d g .
$$

Now, observe that Eq. (4) also applies, in particular, to the Ehresmann connection $\omega_{E}$ in the trivialisation corresponding to $Q_{U}$. Then, comparing Eq. (4) for $\omega_{E}$ with the above Eq. (7) leads to:

$$
\omega_{E}^{U}(x)=\omega_{C}^{U}(x)
$$

\section{B. Levi-Civita coupling from Weitzenböck one-form}

The above considerations show us that, when restricted to the base manifold, both the original Cartan connection and the Ehresmann connection obtained from it, thanks to Sharpe's theorem A.1, are the same on the base manifold, see Eq. (8). They differ mainly through the Maurer-Cartan form between Eqs. (7) and (5). In particular, for the reductive Cartan connection of Eq. (2), the Weitzenböck term remains untouched by the map $\left(\varphi^{*}\right)^{-1}$, which thus cannot lead to a Levi-Civita coupling.

To remedy this problem, one starts by observing that any Ehresmann connection is related to any other by a $G$-invariant ${ }^{7}$ horizontal $\mathfrak{g}$-valued one-form. This can be seen as follows. First, let us consider the difference of any pair of Ehresmann connection one-forms $\omega_{1}$ and $\omega_{2}$. Using Eq. (4) repeatedly for $\omega_{1}$ and $\omega_{2}$, and substracting the result one obtains, in the same local trivialization

$$
\omega_{2}-\omega_{1}=g^{-1}\left(\omega_{2}^{U}-\omega_{1}^{U}\right) g .
$$

This $\mathfrak{g}$-valued one-form is manifestly $G$-invariant and horizontal. Second, if $\alpha$ is a $G$-invariant horizontal $\mathfrak{g}$-valued one-form in $Q$, the sum $\omega+\alpha$, where $\omega$ is an Ehresmann connection, is both $G$-invariant and $\mathfrak{g}$-valued. In addition, since $\alpha$ is horizontal, $\omega+\alpha$ reduces to the MaurerCartan form along fibers. Thus the $\mathfrak{g}$-valued one-form $\omega+\alpha$ is $G$-invariant, and reduces to the Maurer-Cartan form along the fibers, it is consequetly an Ehresmann connection one-form.

This property allows us to recast Eq. (7), up to a redefinition of $\omega_{E}$ under the form

$$
\left(\omega_{E}+\alpha\right)(x, g)=g^{-1}\left(\omega_{C}^{U}\right)(x) g+g^{-1} d g
$$

where $\alpha$ is a $G$-invariant horizontal $\mathfrak{g}$-valued one-form in $Q$. The Eq. (8) in the trivialization corresponding to $Q_{U}$ becomes accordingly

$$
\omega_{E}^{U}(x)+\alpha^{U}(x)=\omega_{C}^{U}(x) .
$$

Then Eq (9) can be recast under the form

$$
\omega_{E}(x, g)=g^{-1}\left(\omega_{C}^{U}-\alpha^{U}\right)(x) g+g^{-1} d g,
$$

Now, let us specialize to our framework in which $P=O M, Q=A M$ and $\omega_{C}=\omega_{W}+\theta$. In that case, one can show [28, theorem 6.2 .5 p. 79$]$ that, for a given Ehresmann one-form $\omega$ on $P=O M$, there exist a unique one-form $\kappa_{\omega}$ on $P=O M$, the so-called contorsion oneform, such that $\omega-\kappa_{\omega}=\omega_{L C}$, the Levi-Civita one form. The contorsion $\kappa_{\omega}$ being thus the difference between two Ehresmann connections it has the properties required to enter in Eq. (10) as the one-form $\alpha$. We can therefore set $\alpha$ to the contorsion corresponding to the Weisenböck connection

$$
\alpha=\kappa_{\omega_{W}}=: \kappa_{W}
$$

in order to obtain the Levi-Civita one-form in the Ehresmann connection $\omega_{E}$ when the Ehresmann part of the

\footnotetext{
7 By $G$-invariance we mean the property for a one-form $\alpha$ to satisfy: $R_{g}^{*} \alpha=A d_{g^{-1}}(\alpha)$, that is for matrix Lie group $R_{g}^{*} \alpha=$ $g^{-1} \alpha g$.
} 
reductive Cartan connection $\omega_{C}$ is $\omega_{W}$. Finally Eq. (10) specializes to

$$
\omega_{E}(x, g)=g^{-1}\left(\left(\omega_{W}+\theta\right)^{U}-\kappa_{W}^{U}\right)(x) g+g^{-1} d g,
$$

\section{The $A M \longrightarrow O M$ map, and the covariant derivative}

In the two previous sections we have shown how, starting from the Cartan-Weitzenböck one-form (2) in $P=O M$, one can obtain the Ehresmann-Levi-Civita one-form $\omega_{E}=\omega_{L C}+\theta$ in $Q=A M$, the principal Poincaré bundle.

As a last step, the covariant derivative, corresponding to the Levi-Civita connection, over the base manifold $M$, can now be obtained thanks to a theorem shown in $[22$, proposition $3.1 \mathrm{p}$. 127] which states ${ }^{8}$ the existence of a map, hereafter $\beta$, which associates to an affine connection, generically $\omega+\phi$ defined on $A M$, the pair $(\omega, \phi)$ on $O M$.

This applies in particular to the affine connection $\omega_{E}$ given in Eq. (11) for which $\beta: \omega_{E} \mapsto\left(\omega_{L C}, \theta\right)$. This map allows us to define the covariant derivative, associated to the reductive Cartan connection (2), as the usual covariant derivative of GR, that is the Fock-Ivanenko derivative. This is the main result of this section.

However, let us emphasize that the Levi-Civita connection appearing in this covariant derivative should be considered as a function of $\omega_{W}, \theta$ and $\eta$ (the Minkowskian metric) these two last quantities entering in the definition of the contorsion $\kappa_{W}$. As a consequence, the LeviCivita connection one-form should not be associated to the gauge field mediating gravity.

\section{VIEWING TEGR AS A GAUGE THEORY OF TRANSLATIONS?}

\section{A. Gauge field vs. connection}

In gauge theories of particle physics, the gauge fields (associated with gauge bosons) are those fields which, at our present energy scale, mediate one of the fundamentals interactions (electromagnetic, weak or strong) between matter fields. They are termed gauge field since their dynamical free equations (uncoupled from matter), involving gauge fields through their field strength, exhibit gauge invariance. On the mathematical side, the gauge fields are recognized to be sections of Ehresmann connections defined on a principal bundle, whose structure group $G$ is a global symmetry group of the free (in the

\footnotetext{
${ }^{8}$ We specialize here this theorem to the sub-bundle of orthonormal frame $O M$ and its affine extension $A M$.
}

sense of uncoupled through gauge fields) matter equations. The field strengths are (sections of) the curvature of these connections one-form. The coupling between a matter field and a gauge field, renders the interacting theory of matter field locally invariant under the symmetry group $G$. Thus, in these theories, on the physical side, the gauge field is a dynamical field which fulfills two related roles: to mediate an interaction and to ensure local invariance under some symmetry.

In classical gravity the spacetime is a metric manifold $(M, g)$, the metric being, in the Cartan view, induced by orthonormal (co-)frames (tetrads) through $\eta(e, e)=g$. This manifold is canonically the base of a frame bundle $F M$. It is a $\operatorname{Gl}(4, \mathbb{R})$ principal bundle which contains the orthonormal bundle $O M$ as a principal $\mathrm{SO}(1,3)$ subbundle. A specific structure, the canonical one-form $\theta$, is always defined on $F M$. It realizes the so-called soldering ${ }^{9}$ and is independent of the existence of any connection on $F M$. In particular, it is worth noting that $\theta$ is not a connection one-form by itself. When an Ehresmann connection is present on $F M$, the canonical one-form allows us to define the torsion. The one-form $\theta$ is specific of the frame bundle, with no equivalent in the mathematical framework of particle physics gauge theory just described, where the Frame bundle, although always defined, is not used. As a consequence, the particle physics framework can be expected to be too restrictive to encompass a gauge theory of gravity involving torsion, such as TEGR.

The above remarks lead us to consider the role played by $\theta$ in our proposal to describe TEGR with the help of the reductive Cartan connection $\omega_{C}=\omega_{W}+\theta$. The canonical one-form appears in two places:

1. in the definition of $\omega_{C}$ Eq. (2), where as a one-form valued in $\mathbb{R}^{4}$, the translation part of the Poincaré algebra, it is identified with the term $\theta$.

2 . in the definition of the contorsion one-form used to rewrite the Levi-Civita one-form as the combination $\omega_{L C}=\omega_{W}-\kappa_{W}$ (see Sec. IV C).

Thus, we observe that the canonical one form $\theta$ is, first, the part of the Cartan connection related to the local translation invariance, and second, enters in an essential way in the coupling with matter. These two characteristics are reminiscent of those retained at the beginning of the present section to identify a gauge field. Since such a field is defined on the base manifold, let us examine the pullback, on the base manifold, of the canonical oneform and its associated Levi-Civita connection, that is the connection that enter the usual gravitational covariant derivative on the base. In the present context, the pullback along some section $\sigma$ of the canonical form $\theta$ reads

$$
\sigma^{*} \theta=e,
$$

\footnotetext{
${ }^{9}$ Note that $\theta$ is not the solder form by itself [see 13].
} 
where $e$ is a local field of frame (a tetrad), and that of the connection $\omega_{L C}$, in some open set $U$ of the base, reads

$$
\omega_{L C}^{U}=\omega_{W}^{U}-\kappa_{W}^{U} .
$$

The Eq. (12), will play a central role in the translationgauge interpretation, because it relates $\theta$, a canonical structure, to the dynamical field $e$. Indeed, Eq. (12) can be read of as the one-to-one relation between $\sigma$ and $e$ induced by $\theta$ : to choose a section is to choose a frame. Now, $e$, in the Cartan view of gravity, is the solution of the gravity field equations. These, are known to exhibit gauge invariance, the choice of a gauge, that is the choice of a particular frame $e$, being, in the fiber bundle context, precisely the choice of a section $\sigma$. These facts point towards the interpretation of the frame $e$ as the gauge field of the theory.

Eq. (13), on another hand can be viewed as the definition of a "fake gauge field", in the sense that the LeviCivita term corresponds to the implementation of the local Lorentz invariance through the covariant derivative, but has no proper dynamics, other than that being induced by the tetrad $e$. Indeed, the first term on the r.h.s. of (13), the Weitzenböck connection one-form, only involves local Lorentz transformations and has a null curvature (field strength), while the second term on the r.h.s. of (13), the contortion one-form, is built on $\eta$, the constant Minkowskian metric, and the field $e$. The tetrad $e$ thus appears here as a dynamical field which "drives" the Lorentz invariance.

We finally come to the conclusion that, at least in the context of TEGR, a distinction should be made between the gauge field and the connection. Note that, such a distinction does not imply any change in particle physics theory (at least at our energy scale), since the structures involved in that extension are not present (not used) in the particle physics framework.

\section{B. A new gauge paradigm for TEGR ?}

This observation, shows us a possible way to interpret TEGR as a gauge theory of translations if we allow one to broaden the structure of a gauge theory by introducing a distinction between the gauge field and the connection. In this view, the gauge field is defined as the pullback on the base manifold, along some section $\sigma$, of the canonical form $\theta$, that is as a frame $e$. To remind the motivations for such an interpretation, let us recall that the field $e$ :

1. is a dynamical field, whose equation exhibits gauge invariance,

2. mediates the interaction through the Levi-Civita connection, Eq. (13), which in the present context is induced by the field $e$, as described at the end of Sec. V A,

3 . is to some extent responsible for the local Lorentz invariance in the sense that the Levi-Civita connec- tion is here determined by $e$ and structural elements as $\eta$.

These three properties are characteristic of a gauge field (Sec. V A). Then, if we insist to interpret TEGR as a gauge theory, we can describe it using $e$ as the gauge field of translations associated with the Cartan connection $\omega_{C}$ Eq. (2).

As explained at the end of Sec. V A, this interpretation does not require any changes in the usual framework of particle physics gauge theories since the departure from that framework relates to extraneous quantities: $\theta, e$. Nevertheless, it requires a distortion of the attributes of the gauge field, in the sense that the field associated to translations, the tetrad $e$, does not implement a local invariance nor mediates the interaction in the same way as gauge field of particle physics would do. This is of course related to the nature of that field, which is not a connection.

Here, the coupling made through the Levi-Civita connection, although induced by the translation field $e$, relates to Lorentz invariance. Furthermore, coupling to matter involves the corresponding representation of the Lorentz group, in particular matter's spin. For a scalar field, as it is spinless, that coupling reduces to zero. Since gravity seems to couple universally to matter, that is independently of its spin, the gravitational coupling should also arise from elsewhere. Indeed, since the generators of the translations span the space $\mathfrak{g} / \mathfrak{h}=\mathbb{R}^{4}$ of the Poincaré algebra and, as seen in Sec. II A, this space is identified to the tangent space of the base manifold, the expansion of the partial derivative operator on the tetrad basis in a neighborhood of some point $x$,

$$
\partial_{\mu}=\left(\partial_{\mu}\right)^{a} e_{a}(x)
$$

shows that the partial derivatives are related to local translations. The universal coupling to gravity should thus be assigned to the partial differential operator, and related to local and infinitesimal translations. We remark that this interpretation is reminiscent of that of the translation-only gauge theory [see 1, sec 5.3], although it avoids the problematic identification of the gauge field with a connection mentioned in the introduction Sec. I.

Here, we point out that, although the structure of the coupling to matter of our Cartan-TEGR formulation is mathematically well defined, the gauge interpretation of the tetrad is more a matter of opinion. The conceptual split between the concepts of gauge field and connection being sound, objections can be raised on the protracted reasoning that leads to the link between translations and the gauge field $e$. In particular, gauging translations in the present context does not correspond to replacing a global symmetry by a local one, the translations generated by the $\mathfrak{g} / \mathfrak{h}$ part of the Poincaré algebra being always local (and infinitesimal). We offer such interpretation to the adhesion from the reader, but abstain from claiming it. 


\section{CONCLUSION}

The main aim of this article is to show that it is possible to retrieve the correct coupling to matter in TEGR starting from a Cartan connection and following a well defined and robust procedure to obtain the familiar LeviCivita form which of course fits all presently available data. To achieve this we use a powerful theorem by Sharpe that yields a one to one correspondence between Cartan connections and Ehresmann affine connections, we apply the Equivalence Principle to extract the LeviCivita connection from TEGR's Weitzenböck one-form, we eventually map the results from the Affine bundle to the Frame bundle. Following these steps the coupling to matter is then given by the usual Fock-Ivanenko covariant derivative appearing as a consequence of the structure descending from the initial choice of Cartan connection.

Beside showing how to coherently retrieve the correct coupling to matter from TEGR with a Cartan connection, we adventure in discussing how the usual paradigm of gauge theories in classical particle physics needs to be enlarged if one insists on interpreting the structure of the TEGR as a gauge theory for the translation group. More precisely, it appears that a distinction between the connection (on spacetime) and the gauge field is required: in such interpretation torsion appears as the field strength of the Cartan connection, justifying the Cartan structure to reproduce TEGR in a bundle framework, and the co-frame (tetrad) as the gauge field related to local translation invariance. We note furthermore that the Lorentz invariance is also driven, although indirectly, by the coframe.

As an interpretation of the theory here described, the gauge theoretic nature of TEGR could certainly still be discussed and adhesion to such interpretation is left to the reader. Nevertheless, the Cartan connection approach gives a new and consistent theoretical description of TEGR.

\section{ACKNOWLEDGEMENTS}

The authors wish to thanks, D. Bennequin for helpful discussions on geometry. The work of M. Le D. has been supported by Lanzhou University starting fund, and the Fundamental Research Funds for the Central Universities (Grant No.lzujbky-2019-25).

\section{Appendix A: Relating Ehresmann and Cartan connections}

For convenience we reproduce here the theorem [Prop. 3.1 p. 365 of 25], relating Ehresmann to Cartan connections, that we use to obtain a covariant derivative.

Let $G$ be a Lie group and $H$ a subgroup of $G$, $P:=P(M, H)$ a principal bundle.
Let $Q:=Q(M, G)=P \times_{H} G$ the principal $G$-bundle associated to $P$ by the action by left multiplication of $H$ on $G$. The principal bundle $P$ is a sub-bundle of the principal bundle $Q$ through the canonical inclusion $p \mapsto\left(p, e_{G}\right)$.

Let $E_{Q}$ be the set of Ehresmann connection $\omega_{E}$ on $Q$ such that $\operatorname{Ker}\left(\omega_{E}\right) \bigcap \varphi_{*}(T P)=0$, and $C_{P}$ the set of $\mathfrak{g}-$ valued Cartan connections on $P$.

Theorem A.1 Let $(G, H)$ be a Klein geometry ${ }^{10}$ and let $P$ and $Q$ be principal $H$ and $G$ bundles, over a manifold $M$, respectively. Assume that $\operatorname{dim} G=\operatorname{dim} P$ and that $\varphi: P \mapsto Q$ is an $H$-bundle map. Then the correspondence $\varphi^{*}: E_{Q} \longrightarrow C_{P}$, is a bijection of sets.

The inverse map of $\varphi^{*}$ is defined as follow: let $\omega_{C}$ a $\mathfrak{g}$-valued Cartan connection on $P(M, H)$, it can be extended to a one-form $j\left(\omega_{C}\right)$ on $P \times G$ defined by the expression:

$$
j\left(\omega_{C}\right):=A d_{g^{-1}} \pi_{P}^{*} \omega_{C}+\pi_{G}^{*} \omega_{G},
$$

where $\omega_{G}$ is the Maurer-Cartan form on $G$, while $\pi_{P}$ and $\pi_{G}$ are the projections on $P$ and $G$ respectively. This one-form on $P \times G$, is proven to be the pull-up, from $Q$ to $P \times G$, of the Ehresmann connection $\omega_{E}$ such that $\varphi^{*}\left(\omega_{E}\right)=\omega_{C}$.

\section{Appendix B: Associated bundle $Q$}

In Ref. [13], we commented on associated bundles, and in particular, on associated vector bundles. Here we recall some facts about associated Lie group bundles in relation to our application of the Sharpe theorem to the Cartan connection in Sec. IV A.

Let us remind from [13, appendix 4] that ${ }^{11} P \times_{H} F$, where $P=P(M, H, \pi)$ is a principal left $H$-bundle and $F$ a $H$-space, is a manifold whose points are the orbits (the equivalence classes) for the right action $R_{H}(p, f) \mapsto$ $R_{H}(p, f):=\left(p h, h^{-1} f\right)$ of $H$ on the product space $P \times F$. The projection map $\pi$ of $P$ induces a projection $\hat{\pi}$ from $P \times_{H} F$ onto the base $M$. The fiber of $P \times_{H} F$ over some $x \in M$ is then defined as $\hat{\pi}^{-1}(x)$. Then, on can show that the local differentiable structure of $P$ ensures that $P \times_{H} F$ is a fiber bundle with base $M$, fiber $F$, and structure group $H$.

In Sharpe theorem, the resulting associated bundle $Q=P \times_{H} G$ is, in fact, a principal $G$-bundle. This is because the Lie group $G$ (the Poincaré group in our particular framework) contains the Lie group $H$ (the Lorentz

10 A Klein geometry is a pair $(G, H)$, where $G$ is a Lie group and $H \subset G$ a closed subgroup such that $G / H$ is connected [see 25, p. 151]

11 We use here the notation $\times_{H}$ instead of $\times_{\rho}$ in coherence with that of Sharpe and [20]. 
group) as a subgroup. Indeed, $Q$ is the so-called $G$ extension of $P$ [see 20, Sec. 5.3.3]. As a consequence, following our notations Sec. IV A, a local trivialisation of $Q=P \times{ }_{H} G$ correspond to the local product $U \times G, U$ being some open set of trivialization, corresponding local coordinates on $Q$ are $(x, g)$.
[1] R. Aldrovandi and J. G. Pereira, Teleparallel Gravity: An Introduction, $\quad$ Vol. 173 (Springer, 2013).

[2] J. W. Maluf and F. F. Faria, Conformally invariant teleparallel theories of gravity, Phys. Rev. D85, 027502 (2012), arXiv:1110.3095 [gr-qc].

[3] J. B. Jiménez, L. Heisenberg, and T. S. Koivisto, The Geometrical Trinity of Gravity, Universe 5, 173 (2019), arXiv:1903.06830 [hep-th].

[4] J. M. Nester and H.-J. Yo, Symmetric teleparallel general relativity, Chin. J. Phys. 37, 113 (1999), arXiv:gr-qc/9809049 [gr-qc].

[5] R. Ferraro and F. Fiorini, Modified teleparallel gravity: Inflation without inflaton, Phys. Rev. D75, 084031 (2007), arXiv:gr-qc/0610067 [gr-qc].

[6] S. Capozziello, R. D'Agostino, and O. Luongo, Extended Gravity Cosmography, Int. J. Mod. Phys. D28, 1930016 (2019), arXiv:1904.01427 [gr-qc].

[7] S. Bahamonde, C. G. Böhmer, and M. Wright, Modified teleparallel theories of gravity, Phys. Rev. D92, 104042 (2015), arXiv:1508.05120 [gr-qc].

[8] S. Bahamonde and S. Capozziello, Noether Symmetry Approach in $f(T, B)$ teleparallel cosmology, Eur. Phys. J. C 77, 107 (2017), arXiv:1612.01299 [gr-qc].

[9] J. Formiga, Conformal teleparallel theories and Weyl geometry, Phys. Rev. D99, 064047 (2019), arXiv:1905.04764 [gr-qc].

[10] K. Bamba, S. D. Odintsov, and D. Sáez-Gómez, Conformal symmetry and accelerating cosmology in teleparallel gravity, Phys. Rev. D88, 084042 (2013), arXiv:1308.5789 [gr-qc].

[11] P. González, S. Reyes, and Y. Vásquez, Teleparallel Equivalent of Lovelock Gravity, Generalizations and Cosmological Applications, JCAP 07, 040, arXiv:1905.07633 [gr-qc].

[12] M. Krššák, R. J. van den Hoogen, J. G. Pereira, C. G. Böhmer, and A. A. Coley, Teleparallel theories of gravity: illuminating a fully invariant approach, Class. Quant. Grav. 36, 183001 (2019), arXiv:1810.12932 [gr-qc].
[13] M. Fontanini, E. Huguet, and M. Le Delliou, Teleparallel gravity equivalent of general relativity as a gauge theory: Translation or Cartan connection?, Phys. Rev. D99, 064006 (2019), arXiv:1811.03810 [gr-qc].

[14] J. G. Pereira and Y. N. Obukhov, Gauge Structure of Teleparallel Gravity, Proceedings, Teleparallel Universes in Salamanca: Salamanca, Spain, November 26-28, 2018, Universe 5, 139 (2019), arXiv:1906.06287 [gr-qc].

[15] M. Le Delliou, E. Huguet, and M. Fontanini, Teleparallel theory as a gauge theory of translations: Remarks and issues, Phys. Rev. D 101, 024059 (2020), arXiv:1910.08471 [gr-qc].

[16] S. Weinberg, Gravitation and Cosmology: Principles and Application (Wiley, New York, NY, 1972).

[17] D. Ivanenko and G. Sardanashvily, The Gauge Treatment of Gravity, Phys. Rept. 94, 1 (1983).

[18] M. Blagojević and F. W. Hehl, eds., Gauge Theories of Gravitation (World Scientific, Singapore, 2013).

[19] Fecko, M., Differential Geometry and Lie Groups for Physicists (Cambridge University Press, 2006).

[20] C. J. Isham, Modern differential geometry for physicists (World Scientific, Singapore, 1999).

[21] M. Nakahara, Geometry, topology and physics, 2nd ed. (CRC press, 2003).

[22] S. Kobayashi and K. Nomizu, Foundations of Differential Geometry, vol 1 (John Willey \& Sons, New York, 1963).

[23] D. K. Wise, MacDowell-mansouri gravity and cartan geometry, Classical and Quantum Gravity 27, 155010 (2010).

[24] G. Catren, Geometric foundations of Cartan gauge gravity, Int. J. Geom. Meth. Mod. Phys. 12, 1530002 (2015), arXiv:1407.7814 [gr-qc].

[25] R. Sharpe, Differential Geometry: Cartan's Generalization of Klein's Erlangen Program (Springer, New York, 1997).

[26] R. P. Geroch, Spinor structure of space-times in general relativity. I, J. Math. Phys. 9, 1739 (1968).

[27] R. P. Geroch, Spinor structure of space-times in general relativity. II, J. Math. Phys. 11, 343 (1970).

[28] D. Bleecker, Gauge theories and variational principles (Dover, 2005). 